\title{
Study of Planetary Systems Around Giant Stars
}

\author{
Matias I. Jones, James S. Jenkins and Patricio Rojo \\ Departamento de Astronomia, Universidad de Chile; Camino El Observatorio 1515, Las Condes, \\ Santiago, Chile
}

\begin{abstract}
More than 450 exoplanets have currently been detected, most of them by the radial velocity (RV) technique. While the majority of exoplanets have been found around main-sequence (MS) FGK stars $(\mathrm{M} \lesssim 1.5 \mathrm{M} \odot)$, only a small fraction $(\sim 10 \%)$ have been discovered orbiting postMS stars. However, such stars are known to host exoplanets and the detection fraction appears to be larger than for solar-type dwarfs. To date $\sim 30$ planets have been found orbiting giant stars which have revealed interesting properties that contrast with the results found for solar-type stars. We are carrying out a RV search for planets around giant stars in the southern hemisphere in order to study different formation scenarios for planets around intermediate-mass stars and the effect of the post-MS evolution of the host stars on the orbits of close-in planets $(a \lesssim 0.6 \mathrm{AU})$.
\end{abstract}

Keywords: Exoplanets; Stellar evolution

PACS: $96.15 . \mathrm{Bc}$

\section{SCIENTIFIC CONTEXT}

To date more than 450 planetary candidates have been detected using the radial velocity (RV) technique. While most of the stars that host detected planets are main-sequence (MS) FGK stars $(\mathrm{M} \lesssim 1.5 \mathrm{M} \odot)$, only a small fraction $(\sim 10 \%)$ are sub-giants or giants. However, evolved stars present an ideal case where to use the RV to search for planets around intermediate-mass stars $(\mathrm{M} \gtrsim 1.5 \mathrm{M} \odot)$ because their are cooler and rotate slower (Schrijver \& Plos 1993, A\&A, 278, 51) than their former MS progenitors and thus present more and narrower lines in their spectra. Also, post-MS stars with B-V $<1.2$ are quite stable and have a relatively low level of jitter (less than $20 \mathrm{~m} / \mathrm{s}$; Hekker et al. 2006, A\&A 454, 943). To date 50 planetary companions around post-MS stars with masses $>1.3 \mathrm{M} \odot$ have been detected and although the sample is limited, interesting results are emerging.

Firstly, there is a lack of close-in orbits companions (with semi-major-axis $\lesssim 0.6 \mathrm{AU}$ ) around giant stars. Figure 1 shows the semi-major-axis distribution for planets orbiting stars more massive than $1.3 \mathrm{M} \odot$ in the MS stage (filled squares), the sub-giant phase (filled triangles) and the red giant branch (RGB; filled circles). As can be seen there is no planet orbiting giant stars closer than $\sim 0.6 \mathrm{AU}$. This observational result might suggests that planets in close-in orbits are destroyed during the evolution of the host stars through the RGB (see e.g. Villaver \& Livio 2009, ApJ, 705, 81). However, the semi-major-axis distribution of planets around subgiants suggests a different formation scenario around stars more massive than $\sim 1.5 \mathrm{M} \odot$ rather than a host star evolutionary effect, since the subgiants star have not expanded enough to destroy planets orbiting further than $\sim 0.1 \mathrm{AU}$ (Johnson et al. 2007, ApJ, 665, 785). A second interesting result is that giant stars harbouring planets do not show a trend toward metal richness (see 


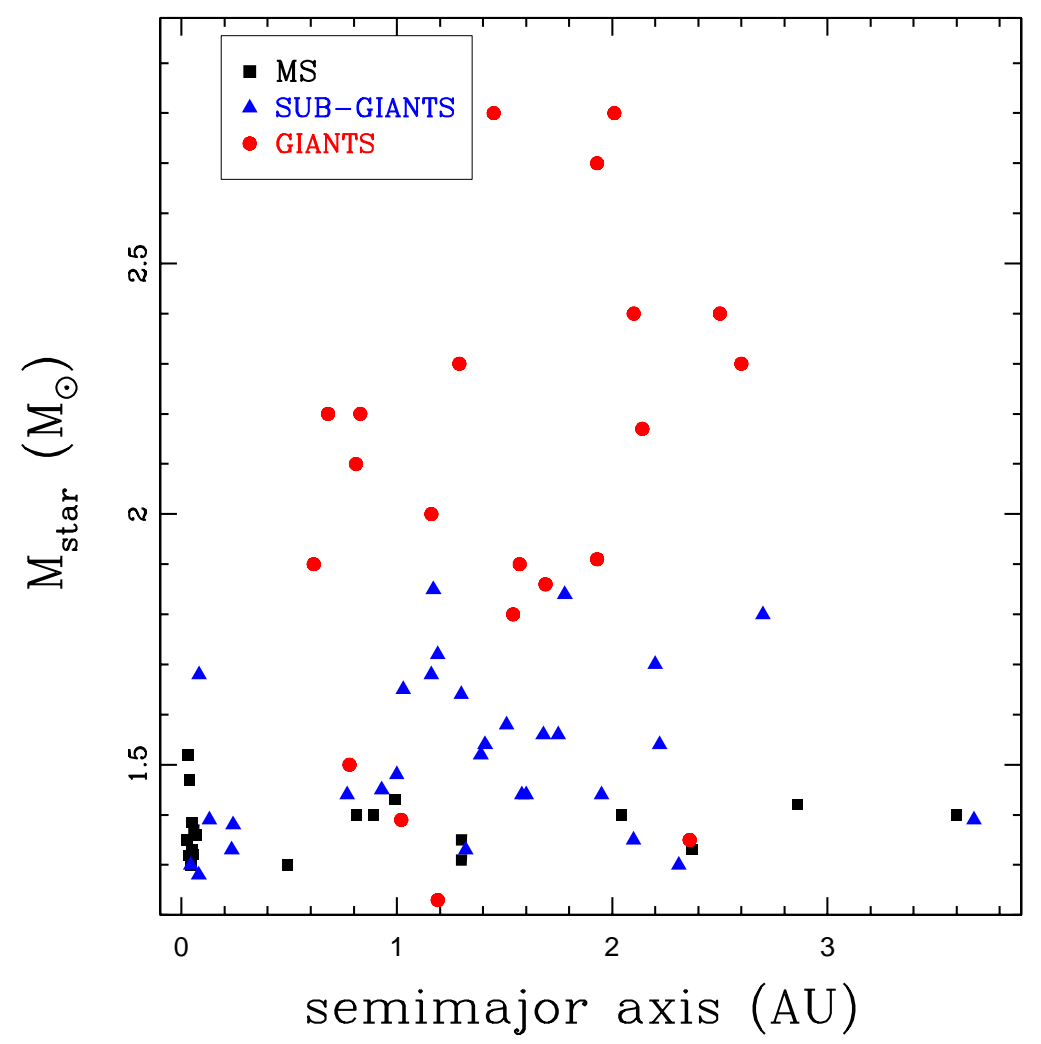

FIGURE 1. Semi-major-axis distribution for planets orbiting stars more massive than $1.3 \mathrm{M} \odot$. The squares, triangles and circles correspond to host stars in the MS, sub-giant phase and giant branch, respectively.

e.g. Doellinger et al. 2009, A\&A, 505, 1311), in direct contrast to what is observed in MS stars (Fischer \& Valenti 2005, ApJ, 622, 1102), which might suggests that the disk instability formation mechanism could be the dominant mechanism by which gas giant planets are formed in massive disks (Boss 2000, ApJ, 536, 101). However, this result could be also be explained as a pollution effect (Pasquini et al. 2007, A\&A, 473, 979). Finally, the fraction of giant stars harbouring giant planets is higher $(\sim 10 \%)$ compared to solar-type stars ( $5 \%$; Doellinger et al. 2009). This result suggests that giant planets are more efficiently formed in massive disks. Clearly a larger census of planets around post-MS stars is required to probe and understand these interesting emerging trends.

\section{TARGETS}

We are monitoring a sample of 150 bright giant stars (most of them brighter than $\mathrm{V}=7.0$ ) in the Southern Hemisphere taken from the HIPPARCOS catalogue, according to their position in the HR diagram (RGB and Horizontal Branch (HB) stars) and precision 


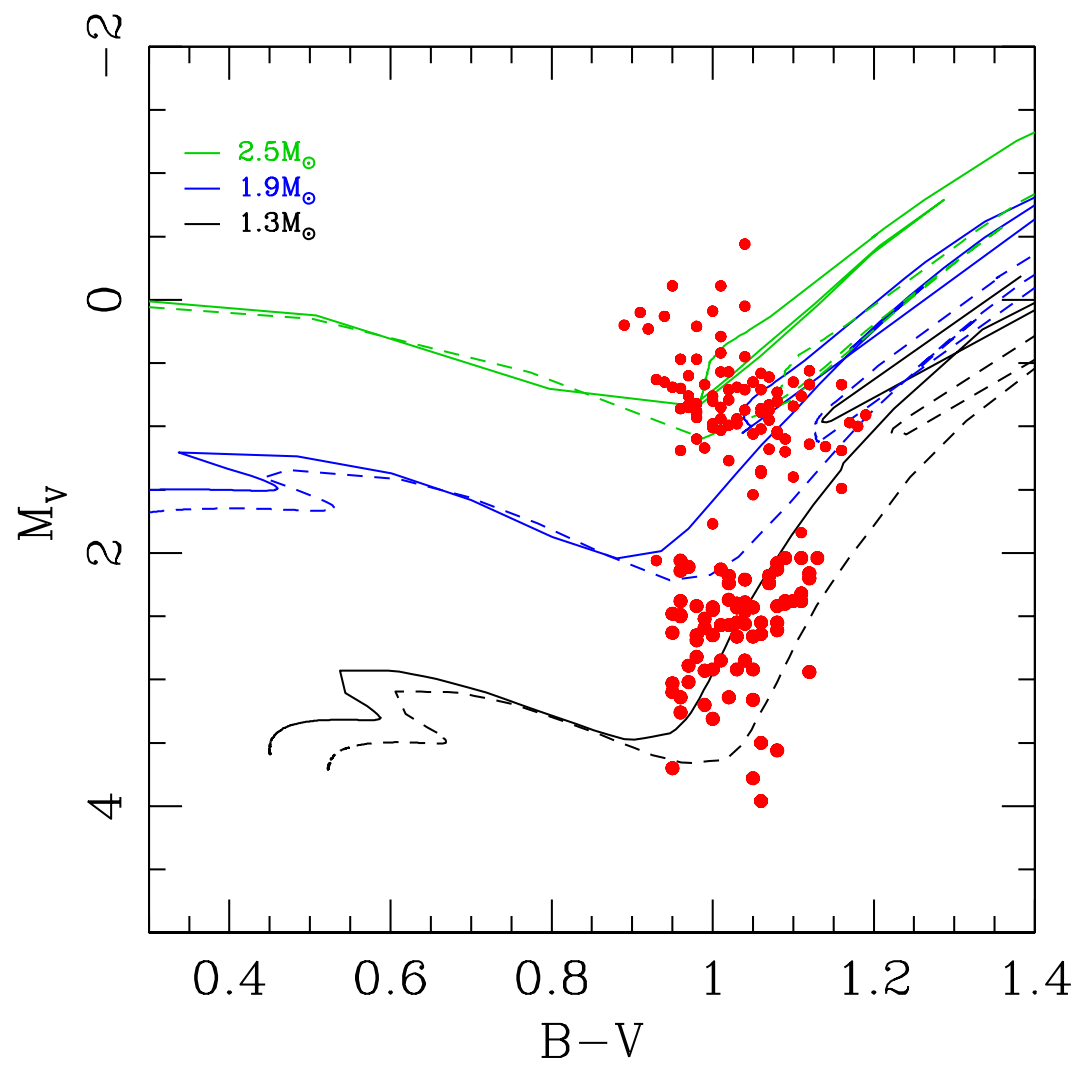

FIGURE 2. H-R diagram including our targets (filled circles). Different evolutionary tracks (Marigo et al. 2008) are overplotted for stars with $1.3 \mathrm{M} \odot, 1.9 \mathrm{M} \odot$ and $2.5 \mathrm{M} \odot$ (line pairs from top to bottom). The solid lines correspond to evolutionary tracks with $[\mathrm{Fe} / \mathrm{H}]=0.0$ and the dashed lines to $[\mathrm{Fe} / \mathrm{H}]=0.2$.

in the parallax (better than $\sim 15 \%$ ). We also removed from the sample stars in binary systems and those with photometric variability greater than $0.015 \mathrm{mag}$. Figure 2 shows the position in the HR diagram for all of our targets (filled red circles). Also in Figure 2 are overplotted evolutionary tracks from Marigo et al. 2008 (A\&A, 482, 883) for stars with ZAMS mass of $1.5 \mathrm{M} \odot$ (black lines), $1.9 \mathrm{M} \odot$ (blue lines) and $2.5 \mathrm{M} \odot$ (green lines). The solid lines correspond to evolutionary tracks with $[\mathrm{Fe} / \mathrm{H}]=0.0$ and the dashed lines with $[\mathrm{Fe} / \mathrm{H}]=0.2$.

\section{CURRENT STATUS OF THE PROJECT}

\section{Observations}

We are currently collecting data using the echelle spectrograph mounted on the $1.5 \mathrm{~m}$ telescope at CTIO, which is equipped with an iodine (I2) cell that can be used to obtain precise wavelength calibrations (Butler et al. 1996, PASP, 108, 500) and therefore 
precise radial velocities. We are also using FEROS, mounted on the $2.2 \mathrm{~m}$ telescope at La Silla Observatory. So far we have taken $\sim 10$ spectra to each of our initial sample ( $\sim 60$ targets) and $\sim 2-6$ spectra for the rest of the targets.

\section{Data Reduction}

We are developing a reduction pipeline (in collaboration with D. Fischer; private communication) for the data taken at CTIO. We are using quartz lamps taken with the I2 cell in the light path, in order to compute the instrumental profile (IP), which is necessary to do the deconvolution of the I2 spectrum from the observed stars spectra. Finally, the radial velocities are computed using a cross-correlation between the observed spectra and a model template.

\section{Atmospheric Parameters}

We have derived atmospheric parameters (effective temperature, surface gravity, microturbulence velocity and iron abundances) for each of the targets in our sample, which are used to determine their masses and ages by fitting evolutionary tracks. These results will be published soon in a paper.

\section{ACKNOWLEDGMENTS}

Matias Jones acknowledges to Alma funding through grant 31080027 and Alma-Sochias for financial support. Patricio Rojo also acknowledges support from FONDECYT, Project 11080271.

\section{REFERENCES}

1. Boss, A. P., ApJ, 536, 101 (2000).

2. Butler, R. P., Marcy, G. W., Williams, E., McCarthy, C., Dosanjh, P., Vogt, S. S., PASP, 108, 500 (1996).

3. Doellinger, M. P., Hatzes, A. P., Pasquini, L., Guenther, E. W., Hartmann, M., A\&A, 505, 1311 (2009).

4. Fischer \& Valenti, ApJ, 622, 1102 (2005).

5. Hekker, S., Reffert, S., Quirrenbach, A., Mitchell, D. S., Fischer, D. A., Marcy, G. W., Butler, R. P., $A \& A, \mathbf{4 5 4}, 943$ (2006).

6. Johnson, J. A., Fischer, D. A., Marcy, G. W., Wright, J. T., Driscoll, P., Butler, R. P., Hekker, S., Reffert, S., Vogt, S. S., ApJ, 665, 785 (2007).

7. Marigo, P., Girardi, L., Bressan, A., Groenewegen, M. A. T., Silva, L., Granato, G. L., A\&A, 482, 883 (2008).

8. Pasquini, L.; Doellinger, M. P., Weiss, A., Girardi, L., Chavero, C., Hatzes, A. P., da Silva, L., Setiawan, J., $A \& A, \mathbf{4 7 3}, 979$ (2007).

9. Schrijver \& Plos, $A \& A, \mathbf{2 7 8}, 51$ (1993).

10. Villaver \& Livio, ApJ, 705, 81 (2009). 